\title{
Opportunities for Energy Storing in Magnetic Field of High-Temperature Superconducting Winding
}

\author{
B. KondratowiCZ-KuCEWICZ \\ Lukasiewicz Research Network - Electrotechnical Institute, \\ M. Pożaryskiego 28, 04-703 Warsaw, Poland \\ Doi: $10.12693 /$ APhysPolA.138.705 \\ *e-mail: b.kondratowicz-kucewicz@iel.pl

\begin{abstract}
The article discusses the possibilities of energy storage in the superconducting winding. Milestones of superconducting magnetic energy storage technologies were presented and the evolution of devices designed in the world was described. This article presents the possible configurations of the hightemperature superconducting winding with particular emphasis on the solenoid and toroidal configuration and the principle of operation of the device. As an example of the device, the design and research results of the Polish superconducting magnetic energy storage physical model with energy $34 \mathrm{~kJ}$ at $13 \mathrm{~K}$ was discussed. The possibility of controlling the energy value and magnetic field distribution using the geometrical parameters of the windings in solenoidal and toroidal configurations was discussed. Studies of the Polish design of the superconducting magnetic energy storage model have shown that energy stored in the superconducting magnetic energy storage winding can be increased. By choosing the appropriate winding configuration with magnetic shielding, a strong magnetic field outside the device can be limited to the values allowed by the standards. Finally, the possible uses of the superconducting magnetic energy storage in the power grid are listed.
\end{abstract}

topics: HTS winding, magnetic field, energy storing, magnetic shielding

\section{Introduction}

Modern power grids are undergoing major changes with increased amount of distributed generation units, such as the solar photovoltaic systems or wind farms. However, the integration of these clean energy sources into the power grids often imposes adverse effects, such as the voltage rise due to a reverse power flow. Various new energy storage technologies to mitigate these problems have been proposed in recent years.

The idea of storing electric energy in the magnetic field of the coil was put forward over 100 years ago, while the use of superconducting materials for these types of devices was proposed in the 1960s. The first demonstration of low-temperature superconductor-superconducting magnetic energy storage (LTS-SMES) with successful charging and discharging was done in 1971 in the University of Wisconsin. The design and development of SMES for utility application was started in 1979. One of the first SMES installations was designed for preventing low frequency oscillations of the Western US Power System by Los Alamos National Laboratory (LANL), USA, and was first commercially installed in 1981. Following this event, many companies started to design and build SMES systems, including Hitachi (1986), Wisconsin Public Service Corporation (2000), ACCEL Instruments $\mathrm{GmbH}(2005)$ and many others but the deployment of SMES has been slow for the last decades due to various restrictions $[1,2]$.

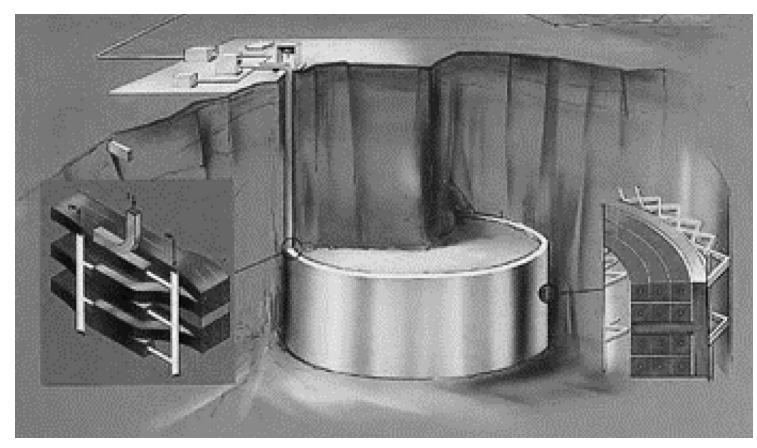

Fig. 1. Representation of one of the first SMESs with two toroidal windings and 106 MJ energy, placed underground in the rock and cooperating with a superconducting DC transmission network $[1,2]$.

The first SMES were designed to accumulate electricity of at least $5000 \mathrm{MWh}$. This idea was to be implemented by constructing solenoid coils from $\mathrm{NbTi}$ with a diameter of $1 \mathrm{~km}$ or several units with a toroidal configuration, to be located underground (Fig. 1). In 1976, the LANL started cooperation with the University of Wisconsin [1,2].

The 30 MJ system developed and installed in 1982-1983 ensured the stability of the Bonneville Power Administration (BPA) which manages a strategic power line called the Pacific AC Intertie (Fig. 2). This example demonstrates the feasibility of the concept of useful energy storage $[1,2]$. 

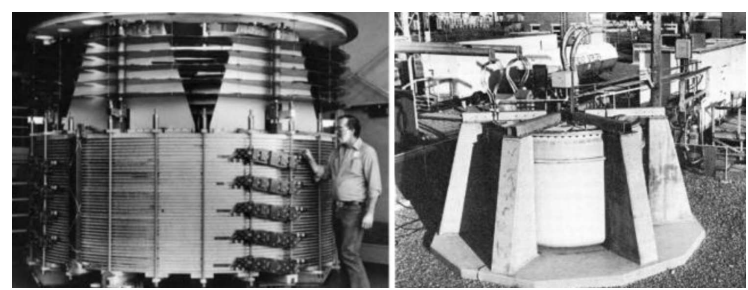

Fig. 2. Electromagnet for 30 MJ SMES made by Los Alamos National Laboratory and installed in Bonneville: $\varnothing_{\text {magnet }}=2.7 \mathrm{~m}$, solenoid winding double pancake, working temperature $=4.5 \mathrm{~K}[1,2]$.

Since the discovery of HTS superconductors, almost all new superconducting devices have been designed on the basis of BSCCO superconductors. The costs of cryogenic cooling have been significantly reduced due to a higher critical temperature of HTS superconductors.

In 1993, the first conceptual design of the HTS BSCCO storage in the USA was created. The constructed device was tested in the energy range from 2 to $200 \mathrm{MW}$ (7.2-720 GJ). A research project was supported by the US Department of Energy. The project conclusions concerned possible current densities in various winding configurations, potential mechanical strains of the winding and magnetic field, and equipment costs. These parameters are still very important today: increasing the density of critical current $J_{c}$, while reducing the cost of HTS material, designing the configuration for higher magnetic field values, increasing the safe range of distortion and minimizing the loss of alternating current. In 1997, American Superconductor announced the results of a $5 \mathrm{~kJ}$ SMES HTS test in $25 \mathrm{~K}$ with a solenoid winding made of a Bi2223 superconductor. It is the first device constructed and successfully tested in the target operating environment — the power grid in Germany. The successful design and testing of SMES has shown that HTS windings could be used in commercial products.

The majority of SMES constructed in the world use a winding made of Bi2223, Bi2212 or YBCO superconductors. There are, however, designs based on the $\mathrm{MgB}_{2}$ superconductor despite of their higher requirements for cooling of windings. The projects and implementations of SMES industrial applications developed in recent years include three groups: $\mu \mathrm{SMES}$ systems with MJ energies entering the commercialization phase, GJ (or MWh) energy systems undergoing design and construction studies and also small energy storage (kilojoules) whose technology still requires intensive research [3].

\section{Operation and the SMES model}

The operation of the superconducting energy store is associated with phenomena that do not exist in conventional energy storage devices. A SMES device is a combination of three technologies: superconductivity, cryogenics and power electronics. Electrically, it is a pure inductor (no internal resistance) and DC current can flow through it without any ohmic loss. As a result, the superconducting coil can make current or energy persistent (1), with the energy density as high as $100 \mathrm{MJ} / \mathrm{m}^{3}$. Though, it charges and discharges very quickly and its discharging time is faster than the charging time. The energy of the magnetic field can be expressed by the dependence [3]:

$$
E=\frac{1}{2} V \mu H^{2}=\frac{1}{2} V \frac{B^{2}}{\mu},
$$

where $H$ is the magnetic field strength, $B$ is the magnetic field induction, $V$ is the volume of the magnetic field, $N$ means the number of turns, $\mu$ - magnetic permeability and $L$ - inductance.

The SMES system consists of a superconducting magnet (to store electric energy), a cooling system CS (to cool the superconducting winding and keep it at operating temperature), a power conversion system PCS (the interface between the AC utility and DC magnet) and a control system [4].

\section{Construction elements of the SMES system}

The electromagnets used in the SMES devices most often have solenoidal configuration but it is not the only possible arrangement. The practical application of SMES entails an issue of efficiency but also an exposure to large magnetic fields.

There is a strong magnetic field for the solenoid winding in a large area around the winding which causes the intensity of the field surrounded by SMES, with high energy values exceeding the limits allowed by the standards, and which is dangerous for users. The superconducting windings are divided into sections and also toroidal configurations are used in many variations (Fig. 3).

In the coil systems or toroids, significant forces act on individual component coils which must be balanced using structural elements [3]. The distribution of these forces depends on the geometry and shape of the windings. To limit the strong magnetic field outside the winding, large SMES devices are now more often designed in a toroidal configuration. However, the energy densities obtained in this configuration are smaller than those in the solenoidal system. In toroidal-modular SMES,
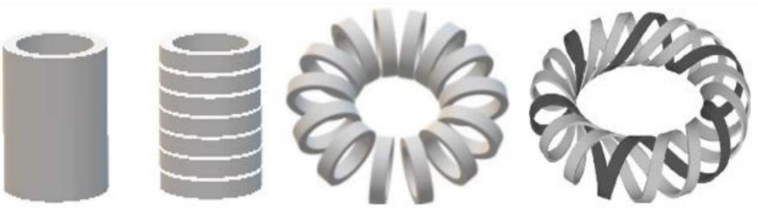

Fig. 3. Possible winding configuration for the SMES system. 
some of the coils have a D shape instead of a circular one - it causes the growth of the energy storage capacity by $18 \%$. However, the cost and complexity would also increase for these designs [3].

The developed constructions are the result of a compromise between the requirement to obtain maximum energy, low costs of the superconducting winding and the necessity to limit the impact of the magnetic field on the environment.

\section{Polish SMES model design and researching}

The superconducting magnet for the SMES model was designed and built in the Lublin Laboratory of Superconducting Technology (Electrotechnical Institute), in cooperation with the Lublin University of Technology as part of the research project entitled "A SMES Device with High-Temperature Electromagnet and Autonomous Cooling System" (Fig. 4) [3, 5]. HTS winding was made of composite tape AMSC Bi-2223 HTS strength wire from American Superconductor Inc. Seven double coils, in which the windings are wound in opposite directions, are placed on a common bobbin (Table I).

The electromagnet operates in a superconducting state at a cryogenic temperature below $77 \mathrm{~K}$ in a cryostat with $7 \times 10^{-6} \mathrm{~Pa}$ vacuum insulation. The model of winding has been experimentally tested under cryogenic conditions for a current in the winding from 25 to $264 \mathrm{~A}$ and a temperature of 77 to $13 \mathrm{~K}$. The results are shown in Fig. 5.

The parameters of SMES magnet.

TABLE I

\begin{tabular}{l|c}
\hline \hline \multicolumn{1}{c|}{ Parameters } & Values \\
\hline pancakes number/turns in pancake & $14 / 140$ \\
outer radius & $0.312 \mathrm{~m}$ \\
height & $0.194 \mathrm{~m}$ \\
length of HTS tape & $1500 \mathrm{~m}$ \\
tape thickness/width & $0.31 / 4.20 \mathrm{~mm}$ \\
$J_{c}$ of tape/77 K & $115 \div 128 \mathrm{~A}$
\end{tabular}

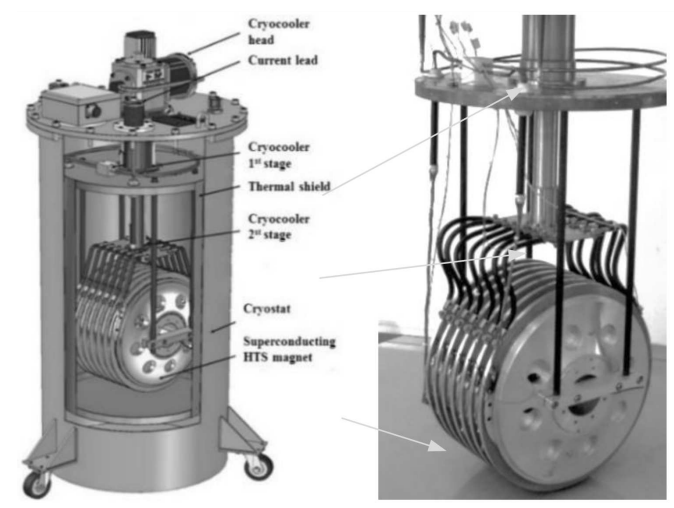

Fig. 4. Project and real model of the HTS SMES magnet $[3,5]$.

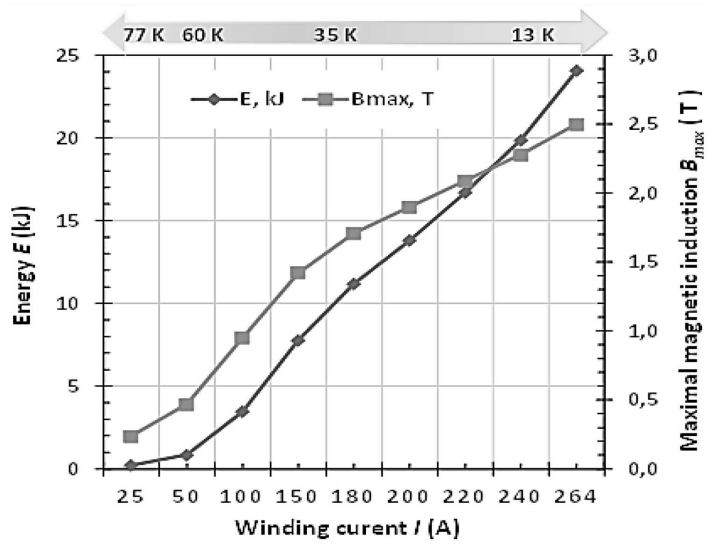

Fig. 5. Energy and magnetic induction in SMES winding depending on current. (a)

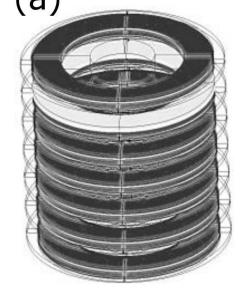

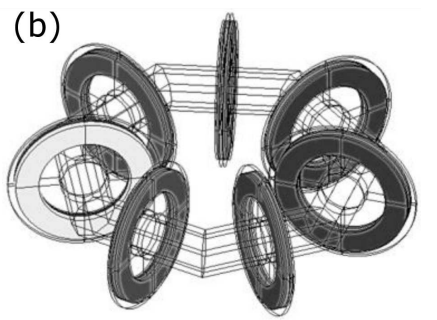

Fig. 6. Superconducting windings for the SMES model in solenoid (a) and toroidal (b) configuration [3].
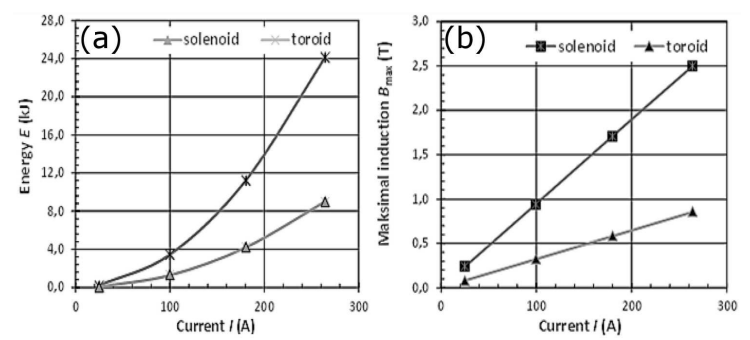

Fig. 7. Dependence of maximum energy stored in solenoid (a) and toroidal (b) configurations.

The magnetic field and energy values depend on the configuration and parameters of the superconducting winding. These dependences were examined in solenoid and toroidal configuration. Figure 6a shows the solenoid configuration used in the physical model. Optional toroidal configuration made of the same windings is presented in Fig. 6b.

Figure 7 shows the dependence of the maximum magnetic induction and energy on the current in the winding in both winding configurations.

The possibility of controlling the value of energy and magnetic field using geometrical parameters of the windings in both configurations was investigated. In the solenoid configuration, the influence of distance between the winding sections on the energy value was determined. In the toroidal configuration, the toroid radius was increased and the magnetic field and energy were studied (Fig. 8). 


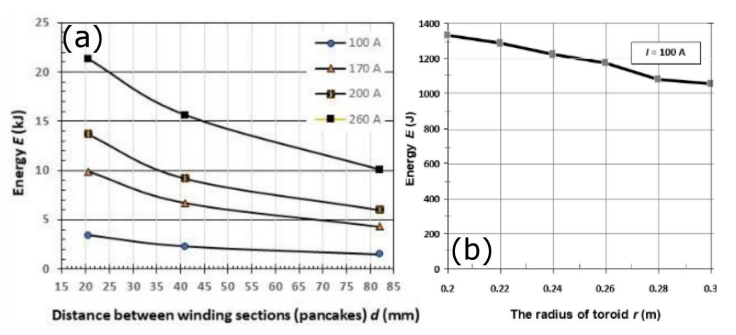

Fig. 8. Energy in SMES winding depending on the distance of pancakes (a) and radius of toroid (b).

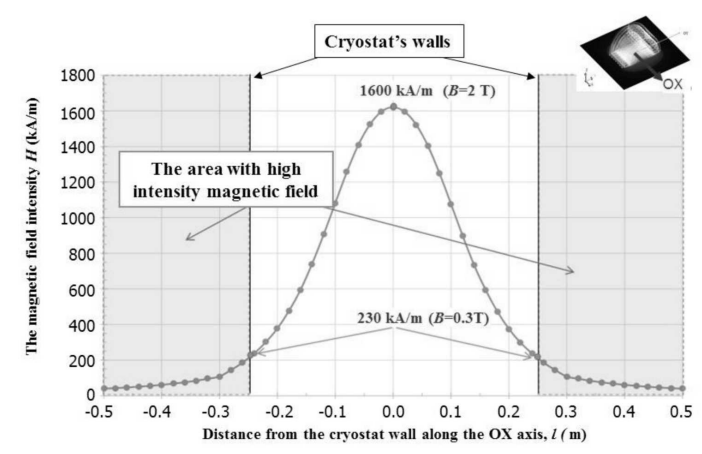

Fig. 9. Magnetic field intensity $H[\mathrm{~A} / \mathrm{m}]$ distribution for operating current $I=264 \mathrm{~A}$ as a function of the distance from the magnet centre [4].

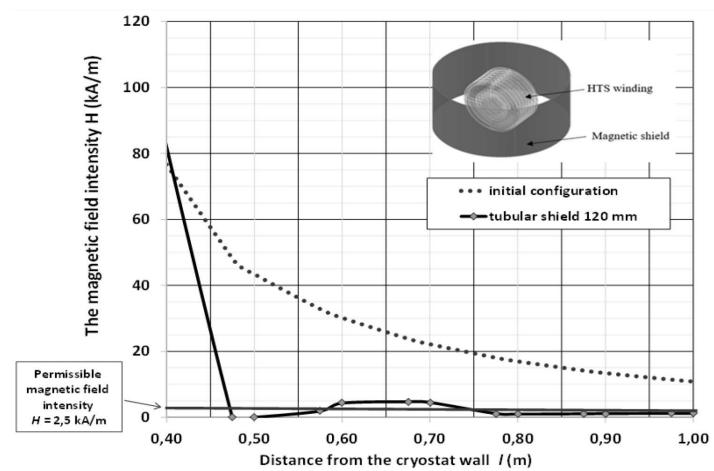

Fig. 10. Magnetic field intensity $H[\mathrm{~A} / \mathrm{m}]$ for tubular shield configuration for $120 \mathrm{~mm}$ shield thicknesses. $I=264$ A [3].

A solenoid configuration was chosen for the SMES HTS model built. It is a compromise solution due to the costs, simplicity of construction and acheived parameters of energy. However, the problem is the value of the magnetic field outside the winding cryostat that exceeds the norm. According to Polish standards, the permissible component of the electromagnetic field in the form of magnetic field strength $H$ is $2.5 \mathrm{kA} / \mathrm{m}$. This value is defined as the acceptable safe value for humans. The value of the field outside the cryostat reaches 100 times higher values and decreases with distance (Fig. 9).

To limit the effect of a strong field, a method of winding shielding with the help of additional ferromagnetic elements has been proposed (Fig. 10).
Simulations of system operation in selected configurations have shown that it is possible to reduce the magnetic field to permissible values while increasing energy from 4 to $13 \%$ for the maximum operating current $264 \mathrm{~A}$.

\section{Possible SMES applications}

SMES installations can be used in power networks for the following applications:

- A GJ-class SMES device is a promising application for load fluctuation compensation in high-voltage power transmission systems;

- SMES installations can improve voltage stability by absorbing excessive power or by discharging it in the grid when there is extra generation (renewable energy sources);

- SMES can provide protection in case of power outage for sensitive loads uninterruptible power supply (UPS);

- Charging stations SMES can be used for storing vast amounts of energy which can be used during periods of decreased generation capacity;

- D-SMES trucks: distributed SMES installations can be loaded on trucks and moved between certain grid points for voltage stabilization or during network failure.

To reduce the capital cost for practical applications and increase the performance of a large-scale SMES, the storage system can be built in combination with other storage system to form a hybrid energy storage system.

\section{Conclusions}

SMES is a promising, highly efficient device. SMES systems have attracted attention of both electric utilities and the military due to their fast response and high efficiency (a charge-discharge efficiency over 95\%). Possible applications include load leveling, voltage stability, frequency regulation, power quality improvement, uninterruptible power supplies, etc. The one major advantage of the SMES coil is that it can discharge large amounts of power in a short time. Also, an unlimited number of charging and discharging cycles can be carried out. Several factors (coil configuration, ability of energy storage, structure and operating temperature) are taken into account in the design of the coil to achieve the best possible performance of an SMES system at the least cost.

Studies of the Polish design of the SMES model have shown that the energy stored in the SMES winding can be increased. By choosing the appropriate winding configuration with magnetic shielding, the strong magnetic field outside the device can be limited to the values allowed by the standards. 
SMES is an attractive solution for commercial applications in the power industry to improve energy quality as well as to cooperate with renewable energy sources. Further work is needed to improve energy storage efficiency and reduce the price of device components such as superconducting winding materials and cryogenic cooling systems.

\section{References}

[1] S. Eckroad, I. Gyuk, EPRI-DOE Handbook of Energy Storage for Transmission \& Distribution Applications, Electric Power Research Institute Inc., 2003, p. 3.
[2] H.J. Boenig, J.F. Hauer, IEEE Trans. Power Appl. Syst. PAS-104, 302 (1985).

[3] B. Kondratowicz-Kucewicz, Prace Instytutu Elektrotechniki 278, 73 (2018).

[4] X.D. Xue, K.W.E. Cheng, D. Sutanto, Ind. Applic. Conf. 2, 1524 (2005).

[5] G. Wojtasiewicz, T. Janowski, S. Kozak, B. Kondratowicz-Kucewicz, J. Kozak, P. Surdacki, B.A. Glowacki, J. Phys. 43, 821 (2006). 